\title{
WSO-UV mission WUVS instrument FUV-UV CCD detectors qualification campaign main results
}

\section{Andrey Shugarov, Mikhail Sachkov, Graham Bruce, Mark Robbins, Andrew Walker, et al.}

Andrey S. Shugarov, Mikhail Sachkov, Graham Bruce, Mark Robbins, Andrew Walker, Nick Waltham, Matthew Clapp, Mike Salter, Gayatri Patel, Sergey Kuzin, Eugene A. Vishnyakov, Alexey S. Kirichenko, Andrey A. Pertsov, Denis V. Ivlyushkin, Pavel S. Zavertkin, Anton D. Nikolenko, "WSO-UV mission WUVS instrument FUV-UV CCD detectors qualification campaign main results," Proc. SPIE 11852, International Conference on Space Optics ICSO 2020, 118525D (11 June 2021); doi: 10.1117/12.2599948

SPIE Event: International Conference on Space Optics - ICSO 2021, 2021, Online Only 


\section{International Conference on Space Optics-ICSO 2020}

Virtual Conference

30 March-2 April 2021

Edited by Bruno Cugny, Zoran Sodnik, and Nikos Karafolas
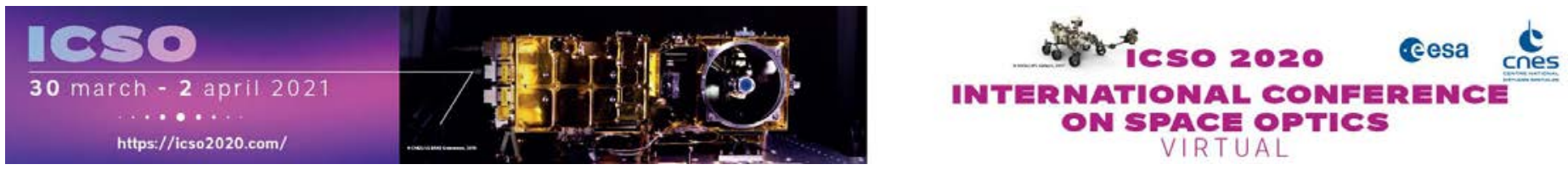

\section{WSO-UV mission WUVS instrument FUV-UV CCD detectors qualification campaign main results}

\section{Cesa isoporeseatings denes}




\title{
WSO-UV mission WUVS instrument FUV-UV CCD detectors qualification campaign main results
}

\author{
Andrey S. Shugarov*a, Mikhail E. Sachkov ${ }^{\mathrm{a}}$, Graham Bruce ${ }^{\mathrm{b}}$, Mark Robbins ${ }^{\mathrm{b}}$, Andrew Walker ${ }^{\mathrm{b}}$, \\ Nick Waltham ${ }^{\mathrm{c}}$, Matthew Clapp ${ }^{\mathrm{c}}$, Mike Salter ${ }^{\mathrm{c}}$, Gayatri Patel ${ }^{\mathrm{c}}$, Sergey Kuzin ${ }^{\mathrm{d}}$, \\ Eugene A. Vishnyakov ${ }^{d}$, Alexey S. Kirichenko ${ }^{d}$, Andrey A. Pertsov ${ }^{d}$, Denis V. Ivlyushkin ${ }^{\mathrm{e}}$, Pavel S. \\ Zavertkin $^{\mathrm{e}}$, Anton D. Nikolenko ${ }^{\mathrm{e}}$ \\ ${ }^{\text {a }}$ Institute of Astronomy of the Russian Academy of Sciences, 48 Pyatnitskaya Street, Moscow \\ 119017, Russian Federation; ${ }^{\mathrm{b}}$ Teledyne UK Limited, 106 Waterhouse Lane, Chelmsford, Essex, \\ CM1 2QU, UK; ' ${ }^{\mathrm{S} T F C}$ Rutherford Appleton Laboratory, Harwell Oxford, Didcot, OX11 0QX, UK; \\ ${ }^{\mathrm{d}}$ P.N.Lebedev Physical Institute of the Russian Academy of Sciences, 53 Leninskiy Prospekt, \\ Moscow 119991, Russian Federation; ${ }^{\mathrm{e}}$ Budker Institute of Nuclear Physics, Russian Academy of \\ Sciences, 11 Ac. Lavrentiev Prospekt, Novosibirsk 630090, Russian Federation
}

\begin{abstract}
World Space Observatory Ultraviolet (WSO-UV) is a major Russian-led international collaboration to develop a large Space-borne $1.7 \mathrm{~m}$ Ritchey-Chrétien telescope and instrumentation to study the Universe in the ultraviolet wavelengths. The WSO-UV WUVS spectrograph consists of three channels: two high resolution channels $(\mathrm{R}=50000)$ with spectral ranges of $115-176 \mathrm{~nm}$ and $174-310 \mathrm{~nm}$, and a low resolution $(\mathrm{R}=1000)$ channel with a spectral range of 115-305 nm. Each of the three channels has an almost identical custom detector consisting of a CCD inside a vacuum Enclosure, and drive electronics with associated cables. The main challenges of the WUVS detectors are to achieve high quantum efficiency in the FUV-NUV range, to provide low readout noise $(\leq 3 \mathrm{e}-$ at $50 \mathrm{kHz})$ and low dark current $(\leq 12$ e/pixel/hour), to operate with integral exposures of up to 10 hours, and to provide good photometric accuracy. Teledyne e2v has designed three variants of a custom CCD272-64 sensor with different UV AR coatings, optimised for each WUVS channel. The custom vacuum Enclosure, also designed by Teledyne e2v, prevents contamination and maintains the CCD at the operating temperature of $-100^{\circ} \mathrm{C}$, while the temperature of the WUVS optical bench is $+20^{\circ} \mathrm{C}$. STFC RAL Space has developed the Camera Electronics Box (CEB) which houses the CCD drive electronics. Digital correlated double sampling technology provides extremely low readout noise and also enables flexibility to optimise readout noise against pixel frequency for a number of normal and binned pixel readout modes. This paper presents the general trade-offs of the WUVS detector design, methods for extending the service life of the CCD sensors working with low signals in a Space radiation environment, and a summary of the measured and calculated key parameters of the WUVS detectors.
\end{abstract}

Keywords: WSO-UV, CCD, quantum efficiency, vacuum ultraviolet, CCD anti-reflection coating.

\section{INTRODUCTION}

The WSO-UV programme ${ }^{1-4}$ aims to study the Universe in the $115 \mathrm{~nm}$ to $310 \mathrm{~nm}$ Ultraviolet (UV) wavelengths. WUVS (WSO-UV UltraViolet Spectrographs) consists of two high-resolution spectrographs $(\mathrm{R}=50000)$ covering the Far-UV range of $115-176 \mathrm{~nm}$ and the Near-UV range of $174-310 \mathrm{~nm}$, and a long-slit spectrograph $(\mathrm{R}=1000)$ covering the wavelength range of $115-305 \mathrm{~nm}$. 


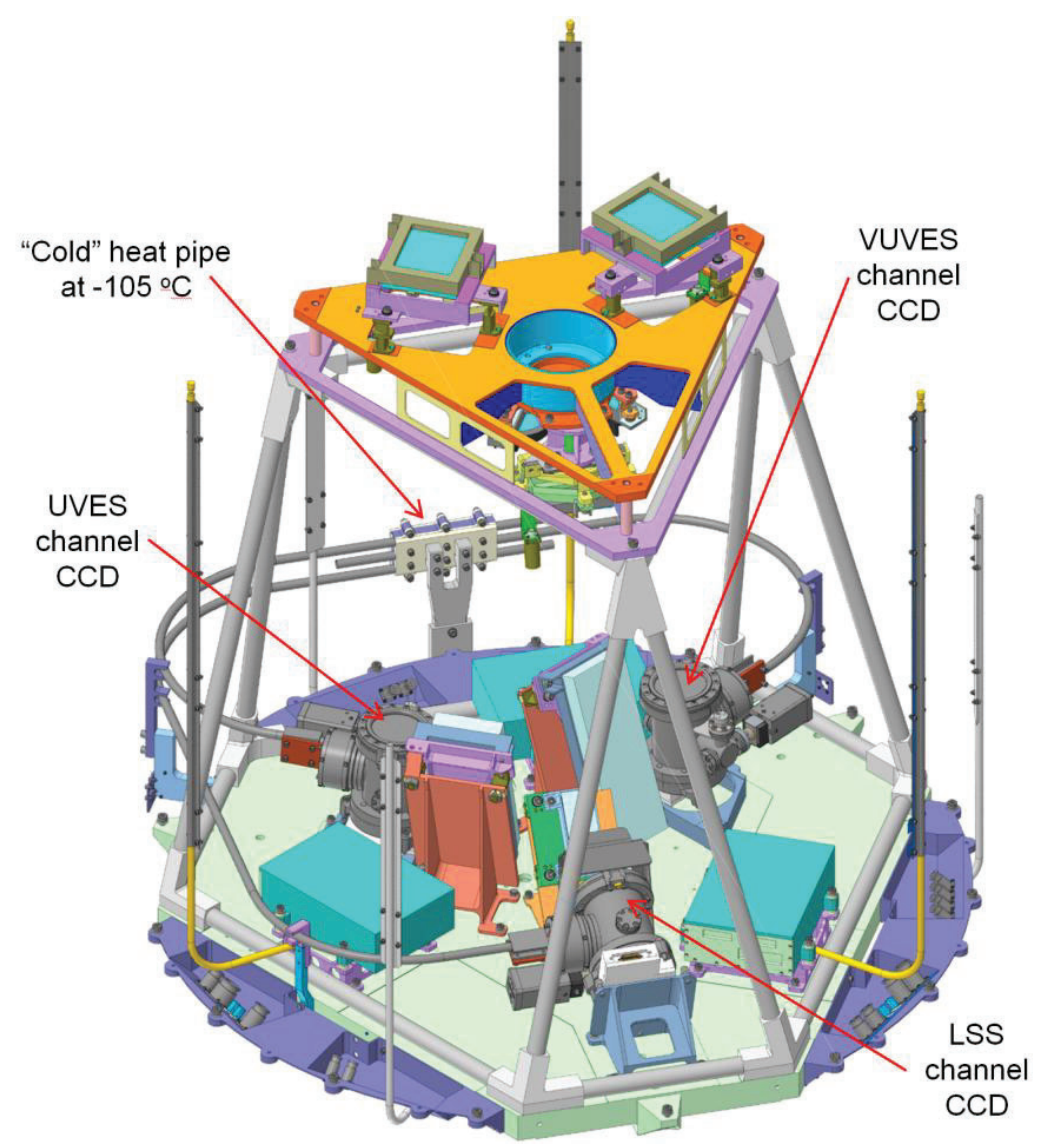

Figure 1. WUVS Spectrograph with 3x CCD detectors.

In the previous version of the WSO-UV spectrographs, it was assumed to use MCP detectors. The disadvantages of the MCP detectors are limited local and global count rates and their lifetime. In the case of using MCP detectors in WUVS, the maximum SNR would be limited at 30 only. The main advantages of the WUVS CCD detectors ${ }^{1,2}$ are high sensitivity in the NUV range, geometrical stability, high dynamical range, possibility to obtain UV spectra with high SNR from relatively bright targets without the risk of damaging the detector. At the shortest wavelength (close to $120 \mathrm{~nm}$ ), the sensitivity of the WUVS CCD system is decreased due to magnesium fluoride window transmittance. In photon-counting mode with a long integration time, an MCP detector can provide better sensitivity for observations of weak sources.

The companies, Teledyne e2v and RAL Space, designed and manufactured the WUVS detector subsystem which consists of three channels, each optimised for a specific range of wavelengths.

The WUVS detectors should provide high sensitivity, high dynamic range and low dark current. For cost-saving reasons the design of all three detectors is identical, except for minor changes such as anti-reflection coating on the CCD and the selection of active output amplifiers.

The Typical WUVS operation scenario of getting the spectrum of a star includes a set of exposures of about 10 minutes each with images co-adding at the science data processing stage on the ground. The total duration of uninterrupted observation can reach 10 hours. During the CCD readout after each sub exposure the light from a star will be blocked by the mechanical shutter. Sub exposures are needed to implement the cosmic ray rejection algorithm and to compensate slow thermal deformations of the WUVS optical system. This shutter will also be used to obtain the dark current calibration images.

Each CCD has an illumination system (a small tungsten lamp with simple optics) which operates in the optical wavelength. The first purpose of this system is to provide a preflash of the CCD at a level of about 10 e-/pixel before 
each sub exposure to fill the traps in CCD. The second purpose is to get the flat field images of a uniform illumination at a level of a few thousand electrons per pixel in optical wavelengths for quick evaluation of the CCD pixel health to exclude bad pixels from the data processing.

For minimisation of the scattered light background in optical wavelengths, all the elements inside the WUVS optical compartment are painted black, and the T-170M telescope optical compartment together with the WUVS optical compartment provides double protection from the direct Sun illumination.

The CCD enclosures and drive electronics are located inside an optical-mechanical unit of the spectrograph (see Fig. 1) which is inside the instrumental compartment of T-170M telescope. Mechanical shutters are located near each of the three spectrographs' entrance slits to block the light during the CCD readout procedure.

The main components of the WUVS CCD sub-system are shown in Fig. 2, the main parameters are presented in Table 1.

Teledyne e $2 \mathrm{v}$ has designed three variants of a custom CCD272-64 sensor with different UV AR coatings, optimised for each WUVS channel. The custom vacuum Enclosure, also designed by Teledyne e2v, prevents contamination and maintains the $\mathrm{CCD}$ at the operating temperature of $-100^{\circ} \mathrm{C}$, while the temperature of the WUVS optical bench is $+20^{\circ} \mathrm{C}$.

STFC RAL Space has developed the Camera Electronics Box (CEB) which houses the CCD drive electronics. Digital correlated double sampling technology provides extremely low readout noise and also enables flexibility to optimise readout noise against pixel frequency for a number of normal and binned pixel readout modes. An Interconnect Module (ICM) is mounted directly to the outside of the CCD enclosure and is linked to a CEB via a short electrical harness. ICM provides the CCD video signal amplification to reduce the system noise and additional filtration of the CCD voltages. All commands and data packets transmitted from/to the spacecraft are carried via primary and redundant SpaceWire links. Primary and redundant power to the sub-system is supplied at $27 \mathrm{~V}$.

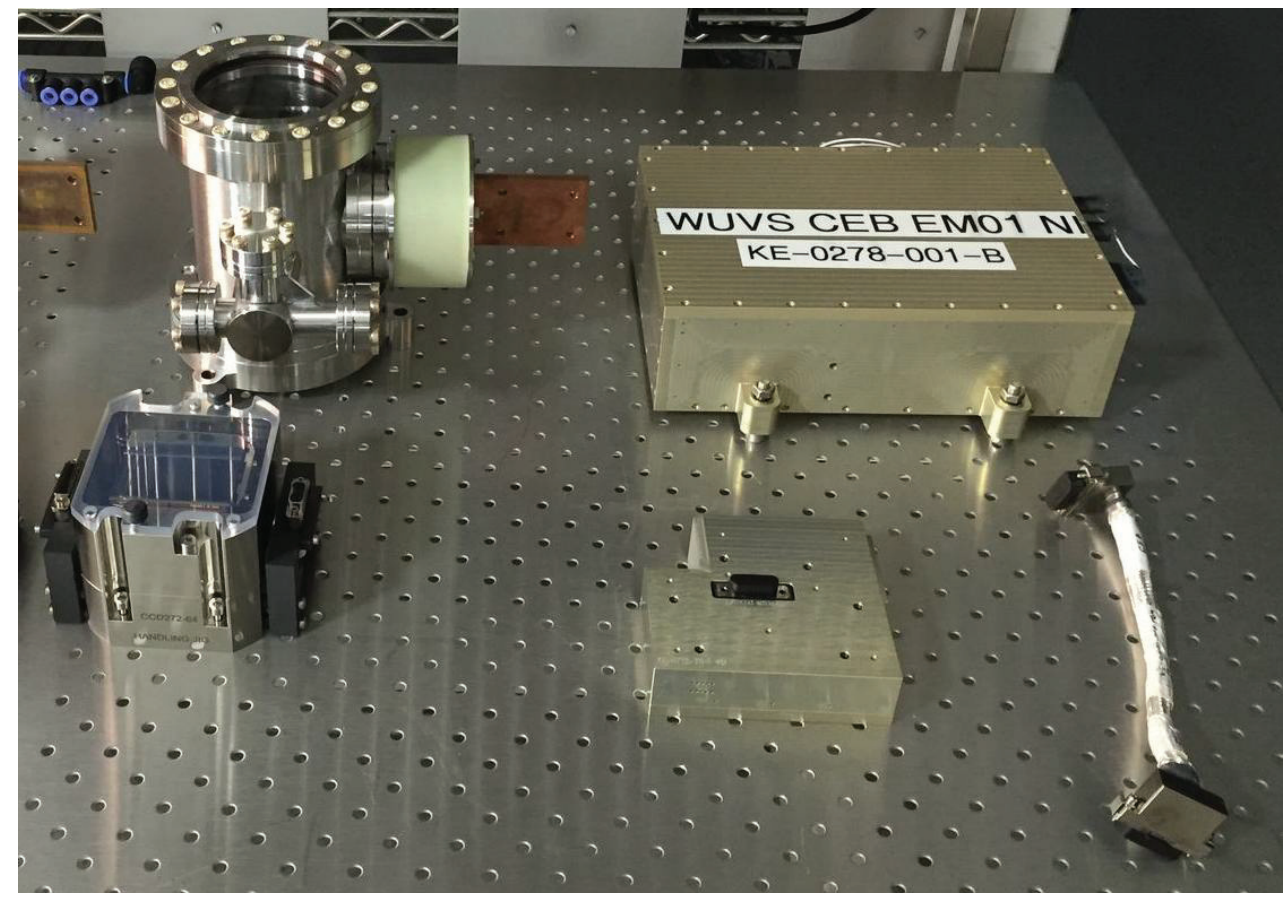

Figure 2. WUVS detector engineering model main parts.

Table 1. Main specifications of the WUVS detectors.

\begin{tabular}{|l|l|l|l|}
\hline Characteristics & VUVES & UVES & LSS \\
\hline
\end{tabular}




\begin{tabular}{|c|c|c|c|}
\hline Spectral range, $\mathrm{nm}$ & $115-176$ & $174-310$ & $115-305$ \\
\hline CCD AR coating & Uncoated & $\begin{array}{l}\text { Uncoated } 174-200 \\
\text { Gradient } 200-310\end{array}$ & $\begin{array}{l}\text { Uncoated 174-200 } \\
\text { Gradient } 200-305\end{array}$ \\
\hline Readout amplifiers & Top, Bottom & Top, Bottom & Left, Right \\
\hline Photosensitive area, $\mathrm{mm}$ & \multicolumn{3}{|c|}{$37.3 \times 49.1$} \\
\hline Pixel format & \multicolumn{3}{|c|}{$4096 \times 3112$} \\
\hline Pixel size, $\mu \mathrm{m}$ & \multicolumn{3}{|c|}{12} \\
\hline Readout speeds, $\mathrm{kHz}$ & \multicolumn{3}{|c|}{$50,100,500$} \\
\hline Readout noise at $50 / 100 \mathrm{kHz}, \mathrm{e}^{-}$ & \multicolumn{3}{|c|}{$3 / 4$} \\
\hline Saturation signal, $\mathrm{e}^{-}$ & \multicolumn{3}{|c|}{30000} \\
\hline Digitalisation, bits & \multicolumn{3}{|c|}{14} \\
\hline $\begin{array}{l}\text { Dark current, } \mathrm{e}^{-} / \mathrm{pixe} / \mathrm{h} \\
\text { at the beginning of life } \\
\text { at the end of life }\end{array}$ & \multicolumn{3}{|c|}{$\begin{array}{l}3 \\
9\end{array}$} \\
\hline $\mathrm{CCD}$ operating temperature, ${ }^{\circ} \mathrm{C}$ & \multicolumn{3}{|c|}{-100} \\
\hline Enclosure foot temperature, ${ }^{\circ} \mathrm{C}$ & \multicolumn{3}{|c|}{+20} \\
\hline Thermal load at Cold finger, W & \multicolumn{3}{|c|}{3} \\
\hline Typical exposure time, $\mathrm{s}$ & \multicolumn{3}{|c|}{600} \\
\hline Data interface & \multicolumn{3}{|c|}{ SpaceWire $25 \mathrm{Mbits} / \mathrm{s}$} \\
\hline Power, V & \multicolumn{3}{|c|}{27} \\
\hline Power consumption, $\mathrm{W}$ & \multicolumn{3}{|c|}{10.5} \\
\hline Mass, kg & \multicolumn{3}{|c|}{9.1} \\
\hline
\end{tabular}

\section{THE WUVS CCD}

Radiation with wavelengths in the range of $100 \mathrm{~nm}$ to $300 \mathrm{~nm}$ is the most difficult to be detected with silicon. This is because the absorption depth is the smallest at these wavelengths. This means that the CCD must be thinned to minimise any dead layer at the back surface.

The WUVS CCD272-64 is a semi-custom version of the device used for the ESA's EUCLID mission. It is a back thinned, back illuminated, 2-phase device pixel array organised by 4096 columns by 3112 rows. The pixel size is $12 \mu \mathrm{m}$ square, but pixels can be combined in $2 \times 2$ groups to give an effective $24 \mu \mathrm{m}$ pixel size.

The optical schemes of VUVES and UVES channels are optimised to use all available areas of the CCD detectors. The spectrum of the LSS channel is located along the bottom long side of the detector. This helps us to minimise the number of parallel transfers during the CCD readout.

The WUVS detectors are optimised to operate with low level signals at $173 \mathrm{~K}$ with a 10 minute standard integration time.

Long-term stability of performance is essential for the success of the WSO-UV project. The radiation degradation of CCD leads to the increasing of the dark current, charge transfer inefficiency and a number of cosmetics defects. To prolong the WUVS CCD detector lifetime in a radiation environment, several techniques are used:

- radiation shielding of CCD by its Enclosure and by other WUVS mechanical elements;

- optimisation of the CCD manufacturing process;

- optimisation of the spectrum layout on CCD to minimise the number of parallel transfers for the LSS channel;

- using split frame transfer CCD to minimise the number of parallel transfers for the VUVES and UVES channels;

- to put the direction of parallel transfers perpendicular to the dispersion in each channel;

- an optical preflash system at a level of about $10 \mathrm{e}^{-}$for partially filling the charge traps; 
- a heating up periodic detector for annealing at a temperature of about $+20^{\circ} \mathrm{C}$.

The two-phase split frame transfer architecture is used to provide maximum charge transfer efficiency after irradiation. The CCD uses a low voltage process to minimise power consumption both on the device and in the drive electronics. To provide the best readout noise characteristics, the conversion factor of output amplifiers is increased and differential output architecture is used to enable common mode noise suppression.

The main challenge for WUVS CCDs is to provide optimised quantum efficiency over a very challenging spectral range from $120 \mathrm{~nm}$ to $320 \mathrm{~nm}$. In order to achieve this, a novel process is used whereby the CCD has an anti-reflection coating over a part of the image area, that varies in thickness to match the required wavelengths. The coating is removed for the shortest wavelengths $(115-180 \mathrm{~nm})$, where the presence of the coating would degrade the quantum efficiency (see Fig. 3).

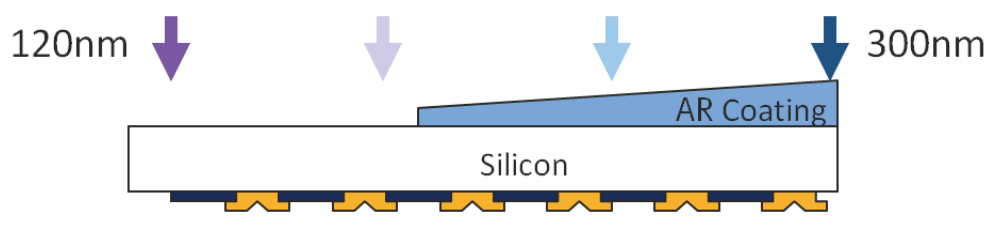

Figure 3. WUVS CCD gradient antireflection coating.

The assessment of quantum efficiency (QE) in the EUV and VUV ranges for modern CCDs is still limited due to the complexity of the necessary equipment, especially the source of monochromatic, well regulated, and uniform EUV and VUV radiations.

In 2019 at the Budker Institute of Nuclear Physics, independent measurements were made of the CCD quantum efficiency in the 115-320 nm range, including a gradient AR coating ${ }^{5}$, and the efficiency of the CCD cooling system. The measurements were carried out at the beamline "Kosmos" using synchrotron radiation from the VEPP-4M storage ring". The beamline utilised a plane grating monochromator configuration. For suppression of the high energy radiation, a $\mathrm{MgF}_{2}$ filter was used. The WUVS CCD Enclosure and the calibrated photodiode SPD (ФДУК-100УВ) were located on a remotely controlled movable optical bench inside the vacuum chamber, to be able to irradiate different areas of the devices and to switch the devices from CCD to SPD and back. We used our experience of previous CCD calibrations ${ }^{7}$. All the measurements were done at the working temperature of $-100^{\circ} \mathrm{C}$ on the sensitive surface of the CCD. Also, the illumination of the CCD provided by the synchrotron, is not uniform, therefore we use diaphragms and a movable optical bench to measure QE at points of interest on the CCD.

We measured QE of the WUVS LSS detector using a complete detector configuration consisting of the engineering qualification model of the Enclosure with CCD installed and a protective $\mathrm{MgF}_{2}$ window, ICM, analog harness and CEB. The LSS device has both an uncoated region (Fig. 4, lower part of the CCD) and a gradient AR coated region. The thickness of the AR coating is optimised for $180 \mathrm{~nm}$ on the left side of the CCD and for $310 \mathrm{~nm}$ on the right side (Fig. 4, upper part of the CCD), in accordance with the WUVS spectrograph dispersion. We measured the QE in the 111-320 nm range for the uncoated region (see Fig. 5). For the AR coated region, we chose 10 points of different thicknesses (see Fig. 6). The resultant measurements show a good compliance with the theoretical predictions made by Teledyne e $2 \mathrm{v}$.

We clearly see a great potential to improve the CCD responsivity in the UV using specially designed anti-reflection coating. For future FUV missions, a new special FUV anti-reflection coating should be designed. 


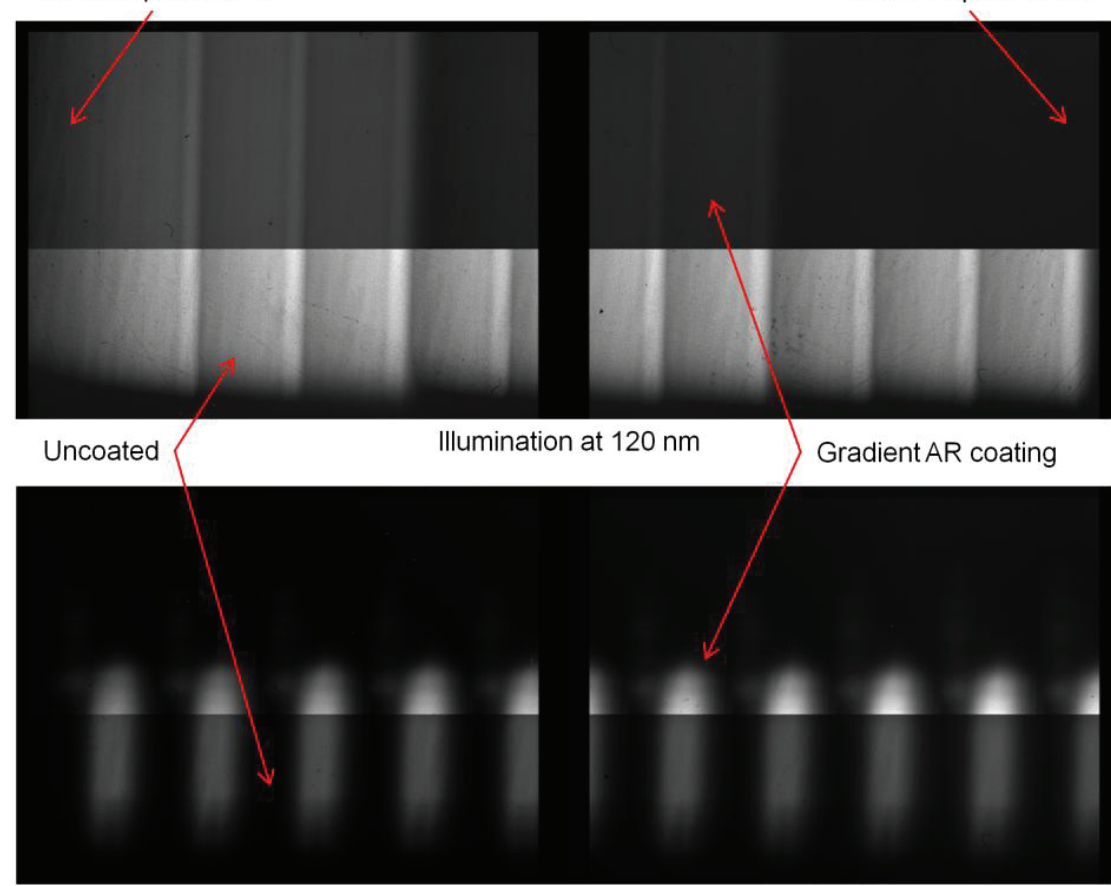

Illumination at $300 \mathrm{~nm}$

Figure 4. EQM LSS CCD test images in FUV and NUV. The lower part of the CCD is uncoated, the upper part has gradient coating.

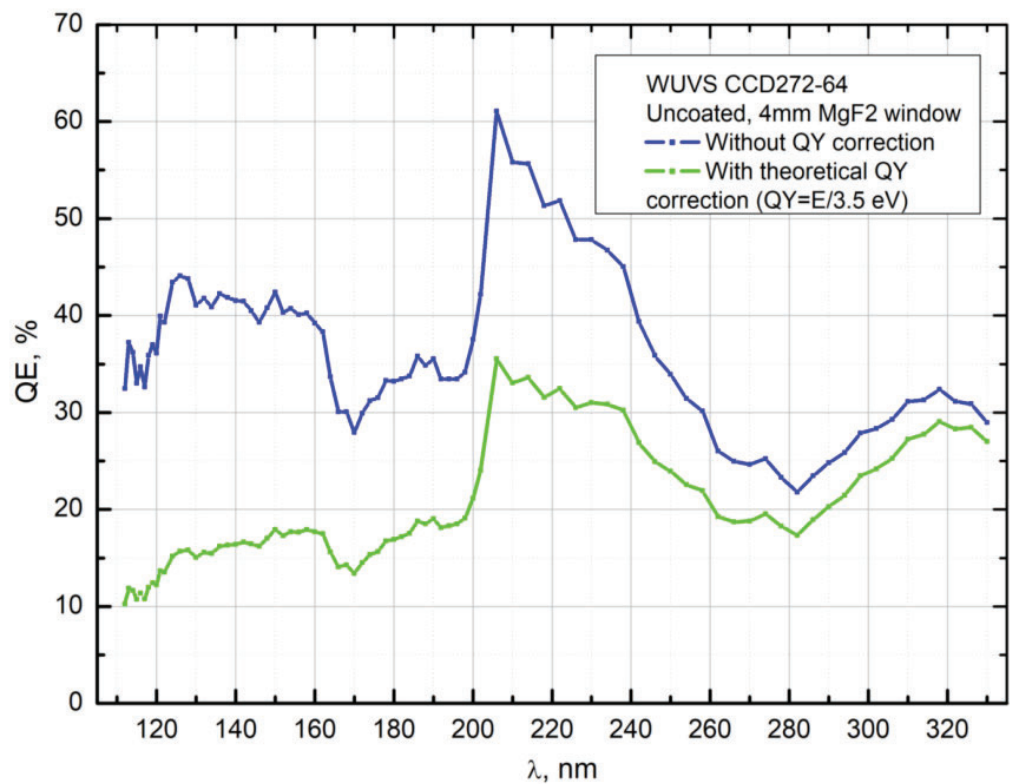

Figure 5. EQM LSS Enclosure quantum efficiency measurement results for the uncoated region of the CCD. 


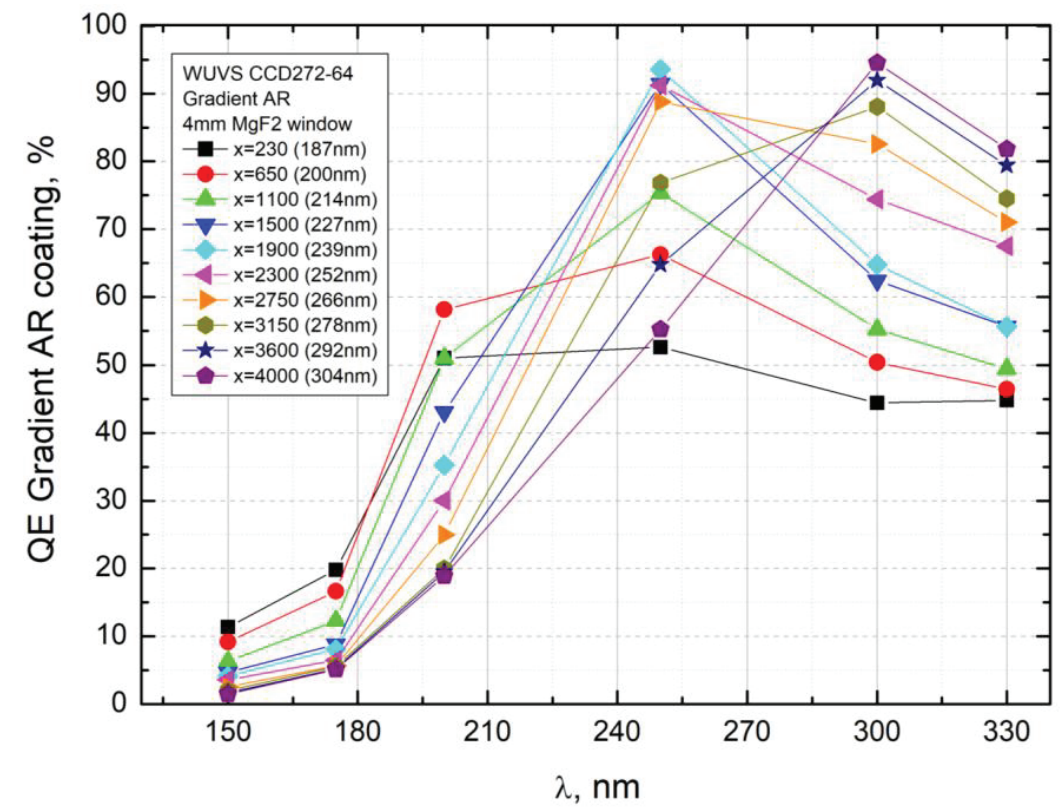

Figure 6. EQM LSS Enclosure quantum efficiency measurement results for the gradient AR coating region of the CCD.

\section{THE HERMETIC CCD ENCLOSURE}

The cold CCD surface is very sensitive to contamination. Teledyne e2v has designed and manufactured a custom cryostat (Enclosure) that can maintain the $\mathrm{CCD}$ at $-100^{\circ} \mathrm{C}$ in ultra-high vacuum conditions over 9 years after delivery.

The purposes of the enclosure are to:

- protect the CCD from mechanical damage in handling, and setup of the system;

- protect the CCD surface from contamination and water condensation;

- provide a structure which can withstand a high vacuum;

- provide an additional radiation shielding for the CCD;

- provide a UV transparent window for illuminating the CCD;

- provide an electrical path for operating the CCD;

- provide a thermal path for cooling the CCD. 

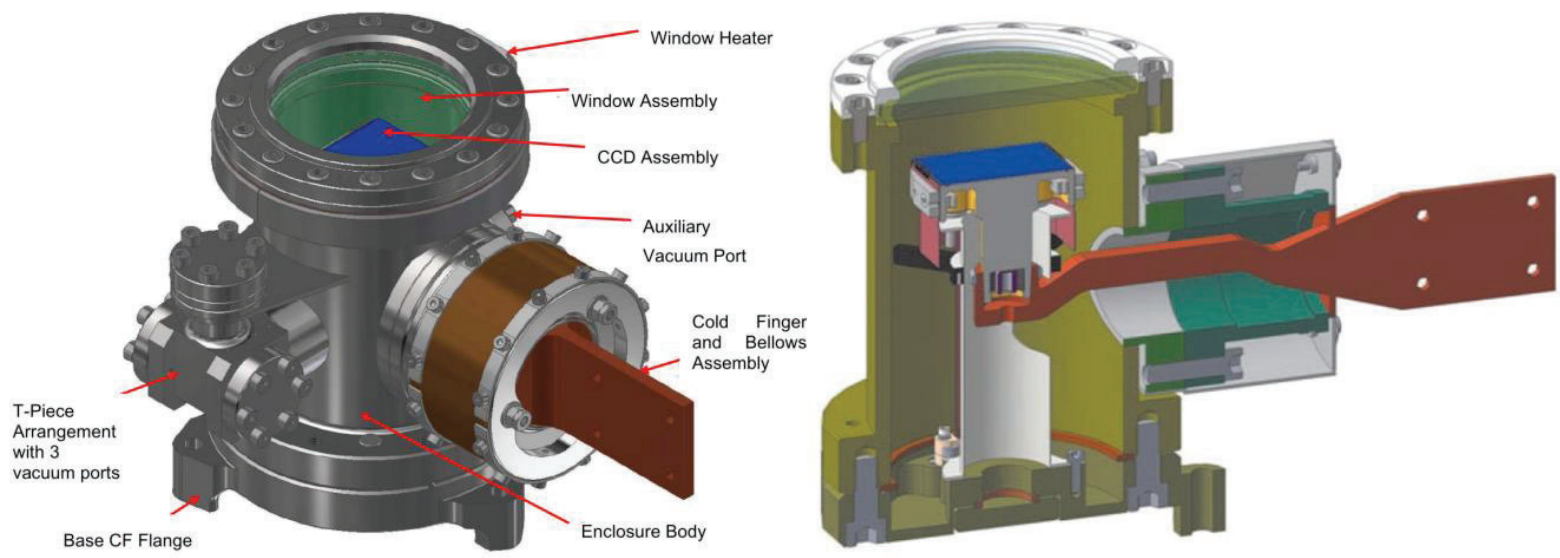

Figure 7. The CCD-containing stainless-steel enclosure with $\mathrm{MgF}_{2}$ protective window and $\mathrm{Cu}$ Cold Finger.
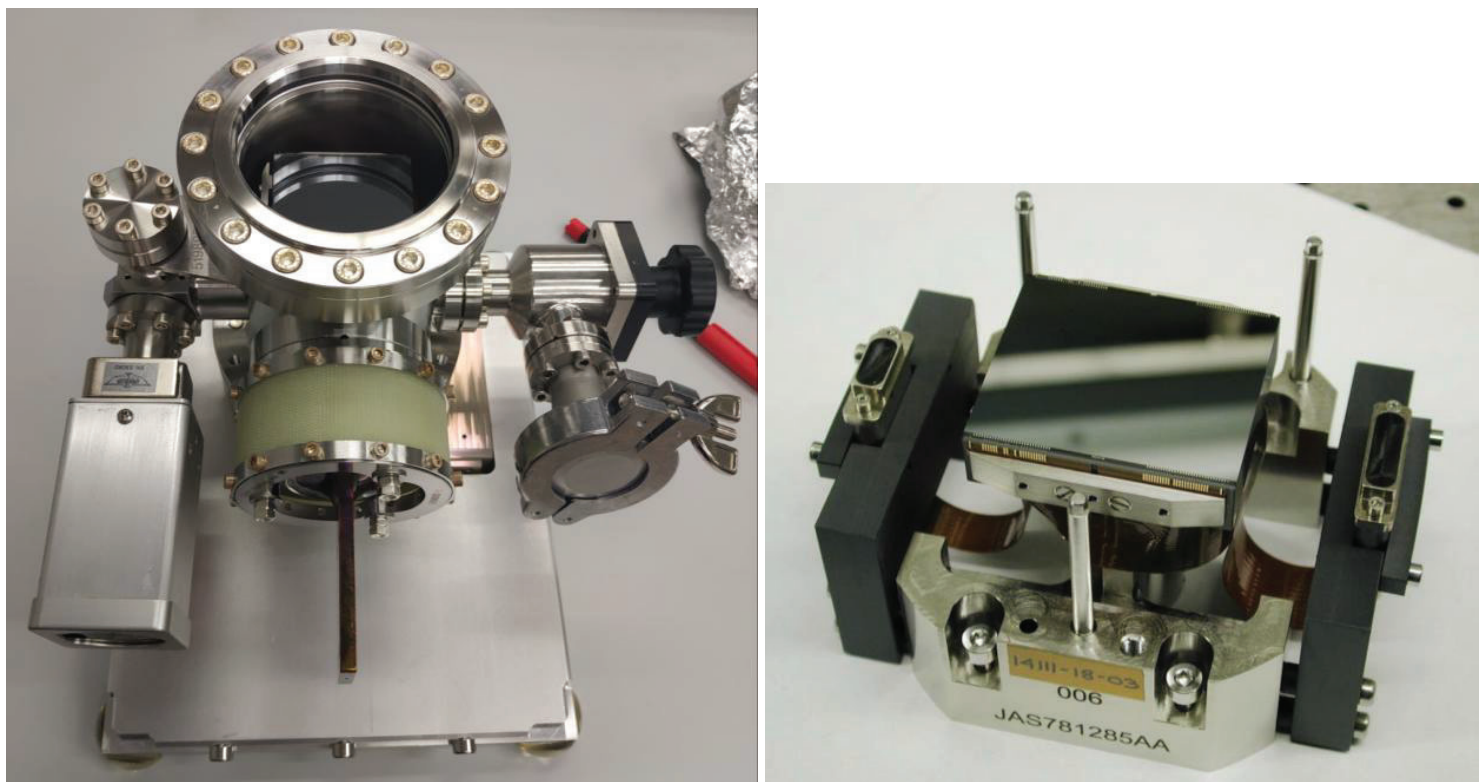

Figure 8. The engineering qualification model (EQM) of the Enclosure with CCD installed (left), the stand alone CCD (right)

Inside the cryostat, only special low-gassing materials were used. Each Enclosure has a $\mathrm{MgF}_{2}$ window and the main body is made of austenitic stainless steel. The central duplex steel column holds the CCD sensor.

The thermal path for cooling the $\mathrm{CCD}$ to $-100^{\circ} \mathrm{C}$ is managed by a high conductivity thermal connection to a heat-pipe on one of the inputs of the chamber. This has been modeled in detail to ensure that the total temperature difference between the input and the CCD is less than $5^{\circ} \mathrm{C}$.

The surrounding temperature inside the WUVS optical compartment is $+20^{\circ} \mathrm{C}$. The Enclosure is mounted on the platform with an active thermal stabilisation system that provides $+20^{\circ} \mathrm{C}$ at the Enclosure's mounting feet.

The WSO-UV spacecraft provides an isolated cold heat pipe connected with a large external radiator. This system maintains the temperature of the CCD Enclosure's "Cold finger" around $-105^{\circ} \mathrm{C}$. A low-power heater is installed near the CCD for precise temperature stabilisation. The measured flatness of the CCD is less than $10 \mu \mathrm{m}$ peak to valley. 
To prolong the detectors' lifetime in a radiation environment, we plan to heat up the CCDs for annealing, from the operation temperature up to the room temperature for a few hours every few months. For this purpose, a $500 \mathrm{~W}$ heater is installed on the external radiator, and an additional $10 \mathrm{~W}$ heater is installed on the Enclosure's cold finger.

The Enclosure input window is heated up to $+22^{\circ} \mathrm{C}$ by an additional dedicated heater to prevent contamination of the window from the side of the WUVS optical compartment. This system is powered directly by the spacecraft battery, to ensure the protection of the CCD window against contamination just after the launch, when WUVS remains unpowered, while the CCD cooling system, which is a passive one, begins to operate.

The results of the Enclosure cooling system verification confirm that the temperature difference between the CCD and the "Cold finger" is $5^{\circ} \mathrm{C}$ (see Fig. 9).

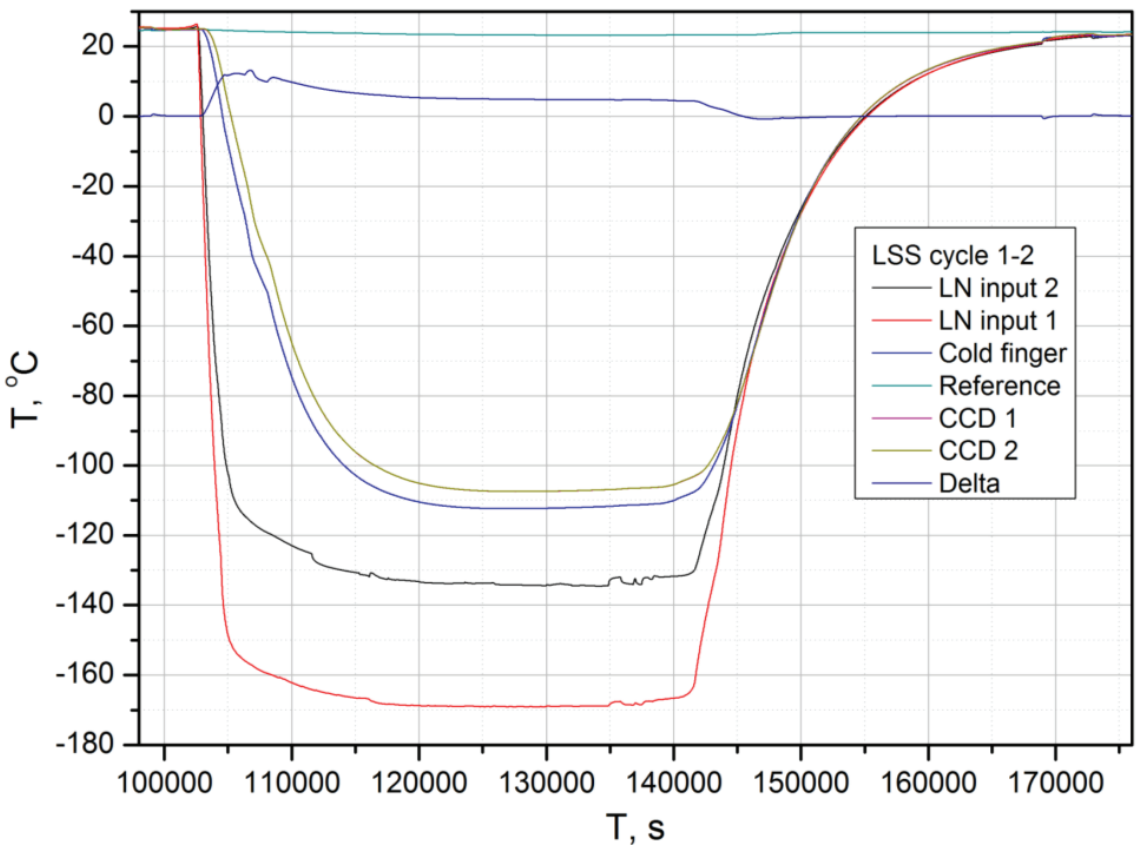

Figure 9. EQM Enclosure cooling system verification (INP, Russia).

\section{CCD DRIVE ELECTRONICS}

The Camera Electronics Box (CEB) was designed and built by RAL Space, STFC (UK) based on its heritage of the production of CCD camera electronics for several projects such as STEREO SCIP/HI, SDO AIA/HMI, GOES-R SUVI.

The main requirements of WUVS CEB are:

- to drive the Teledyne e2v CCD272-64;

- to read out CCD through the two differential amplifiers;

- the video signal digitised to 14 bits with Digital Correlated Double Sampling;

- the readout noise is $<3 \mathrm{e}^{-} \mathrm{rms}$ at $50 \mathrm{kHz}$;

- the programming, control and data transfer over a SpaceWire link (redundant) running at $25 \mathrm{Mbit} / \mathrm{s}$. 

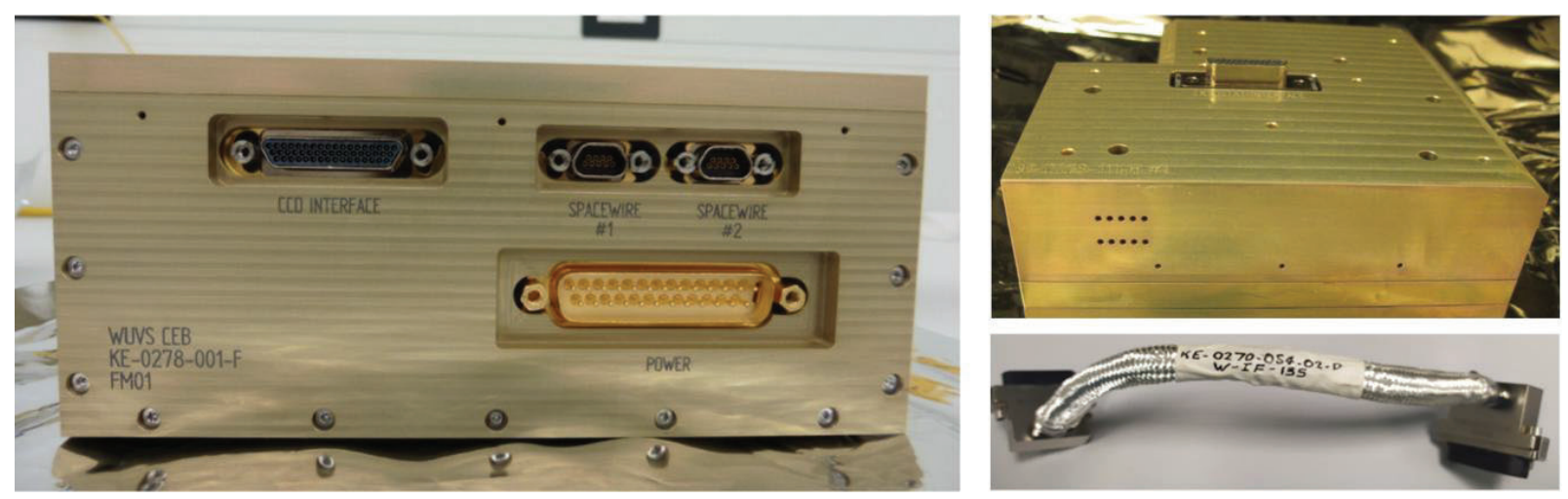

Figure 10. Flight models of the Camera Electronic Box (CEB), Interconnection module (ICM) and analog video cable.

Three identical CEBs are located inside the optical-mechanical unit of WUVS close to the dedicated CCD enclosures to minimise the analog cable lengths. The heat produced by each CEB is evacuated by their own dedicated heat pipe. The CEB houses three camera electronics cards: power supply card, bridge card and CCD camera card (see Fig. 10). The CCD camera card provides the majority of the functionality within the CEB system, including the SpaceWire interface, video digitisation, CCD bias voltage generation and CCD clock drivers. The CCD camera card contains two DCDS (Digital Correlated Double Sampling) video channels that provide the Correlated Double Sampling (CDS) and Analogue-to-Digital Conversion (ADC) which are required to sample and digitise the video signals from the CCD's two readout amplifiers. The ADCs run in parallel at $25 \mathrm{Mpixel} / \mathrm{s}$ and provide 14-bit digitisation.

Differentiating the real CCD output with that of a dummy output (same circuit, but no input) cancels pick-up and reset feedthrough, rejecting system noise and clock-feed through, but with read noise increased by a factor $\sqrt{2}$. In DCDS oversampling of the video waveform gives a total of $\sim 160$ pixel samples at $50 \mathrm{kpixel} / \mathrm{s}$. This reduces the noise by the square root of the number of samples.

The CCD camera card provides a variable pixel readout rate of $50 \mathrm{kHz}, 100 \mathrm{kHz}$ and $500 \mathrm{kHz}$, multiple windowed readout and on-chip pixel-summing modes.

During the RAL Space factory test of the FM CEB, the readout noise of about $2.6 \mathrm{e}^{-} \mathrm{rms}$ at $50 \mathrm{kHz}$ and about $3 \mathrm{e}^{-} \mathrm{rms}$ at $100 \mathrm{kHz}$ were measured. The linearity is better than $0.2 \%$, the cross talk between the channels is less than $29 \mathrm{ppm}$.

\section{SUMMARY AND THE CURRENT STATUS}

The detectors for the WUVS spectrograph have been developed based on the Teledyne e2v and RAL Space experience and consider the specific requirements of the WSO-UV mission to operate with very low signals at a long exposure time.

The Critical Design Reviews (CDR) of the CEB and Enclosure were held in 2016 and 2019 respectively. The WUVS detector subsystem successfully passed the qualification campaign and the key parameters were verified.

In 2019 4x CEB FM (Camera Electronics Box Flight Model) units were successfully delivered to Russia and passed incoming inspection.

3x EQM Enclosures with 3 different CCDs (VUVES, UVES, LSS) were delivered to LPI and passed incoming inspection. In addition to the Teledyne e2v factory test, the quantum efficiency of the LSS EQM Enclosure and the CCD cooling system efficiency were measured in Russia.

The most complicated unit of the system is the Flight model of the Enclosure, which is expected to be delivered at the end of 2022. 


\section{ACKNOWLEDGEMENTS}

The development of the WUVS detectors would have not been possible without the significant contributions made by all members of the WUVS project team.

We express our gratitude to the Teledyne e2v and RAL Space companies for the production of the custom CCD272-64, custom enclosures and low readout noise electronics for the WSO-UV space project.

In addition, special thanks are due to the BINP team for the provision of the facilities used during the testing and characterisation of the CCD in UV and for their continued project support.

\section{REFERENCES}

[1] Shugarov, A., Savanov, I., Sachkov, M., Jerram, P., Moody, I., Pool, P., Turner, P., Pittock, R., Kuzin, S. and Waltham, N., "UV detectors for spectrographs of WSO-UV project," Astrophys. Space Sci. 354, 169-175 (2014).

[2] Hayes-Thakore, C., Spark, S., Pool, P., Walker, A., Clapp, M., Waltham, N. and Shugarov, A., "Sensor system development for the WSO-UV (World Space Observatory-Ultraviolet) space-based astronomical telescope," Proc. SPIE 9639, 96390U (2015).

[3] Sachkov, M., Shustov, B. and Gómez de Castro, A. I., "WSO-UV project," Adv. Space Res. 53 (6), 990-995 (2014).

[4] Shustov, B., Gómez de Castro, A. I., Sachkov, M., Moisheev, A., Kanev, A., López-Santiago, J., Malkov, O., Nasonov, D., Belén Perea, G., Sánchez, N., Savanov, I., Shugarov, A., Sichevskiy, S., Vlasenko, O. and Yaňez, J., "WSO-UV progress and expectations," Astrophys. Space Sci. 354, 155-161 (2014).

[5] Vishnyakov, E. A., Shugarov, A. S., Ivlyushkin, D. V., Zavertkin, P. S., Nikolenko, A. D., Chervinskiy, V. I., Pertsov, A. A., Erkhova, N. F., and Kuzin, S. V., "Evaluation of CCD detector absolute responsivity with the aid of synchrotron radiation," AIP Conf. Proc. 2299, 060007 (2020).

[6] Nikolenko, A. D., Avakyan, S. V., Afanas'ev, I. M., Voronin, N. A., Kovalenko, N. V., Legkodymov, A. A., Lyakh, V. V. and Pindyurin, V. F., "Kosmos Station: Application of Synchrotron Radiation from the VEPP-4M Storage Ring for Metrological Measurements in the VUV and Soft X-ray Ranges," J. Surf. Inv. X-Ray, Synchr. Neutr. Techn. 6 (3), 388-393 (2012).

[7] Vishnyakov, E. A., Shcherbakov, A. V., Pertsov, A. A., Polkovnikov, V. N., Pestov, A. E., Pariev, D. E. and Chkhalo, N. I., "High-aperture monochromator-reflectometer and its usefulness for CCD calibration," Proc. SPIE 10235, EUV and X-ray Optics: Synergy between Laboratory and Space V, 102350W (2017). 